\title{
A rare case of laryngeal tuberculosis simulating malignancy
}

\author{
Ansari S, ${ }^{1 *}$ Amanullah MF, ${ }^{2}$ Ahmad $K,{ }^{1}$ Dhungel K, ${ }^{1}$ Gupta MK, ${ }^{1}$ Kumar $A^{1}$ \\ ${ }^{1}$ Department of Radiodiagnosis and Imaging, ${ }^{2}$ Department of Orthopedics, \\ B.P. Koirala Institute of Health Sciences, Dharan, Nepal
}

\author{
*Corresponding Author: \\ Dr. Sajid Ansari, \\ MD, Department of Radiodiagnosis and imaging, \\ B.P. Koirala Institute of Health Sciences, Dharan, Nepal. \\ Address: H-2733, BPKIHS, Dharan, Nepal. \\ Mobile no.: 00977-9722541028 \\ Email address: drsajidansari@yahoo.co.in

\section{Citation} \\ Ansari S, Amanullah MF, Ahmad K, Dhungel K, Gupta \\ MK, Kumar A. A rare case of laryngeal tuberculosis \\ simulating malignancy. Nepal Journal of Medical \\ Sciences 2013;2(2):200-2.
}

\begin{abstract}
Laryngeal tuberculosis is an uncommon disease and should be considered as a differential diagnosis in any laryngeal disease, in particular in the case of a laryngeal carcinoma. It is difficult to differentiate laryngeal tuberculosis from carcinoma through imaging and needs clinical and histopathological correlation. We describe the case of a 26 year old man who presented with the clinical picture of laryngeal cancer, but which turned out to be tuberculosis.
\end{abstract}

Keywords: Computed tomography ; laryngeal tuberculosis; Malignancy

\section{Introduction:}

Laryngeal tuberculosis (TB) is a rare disease and it is seen in nearly $1 \%$ of cases of tuberculosis. ${ }^{1-3}$ Imaging features of laryngeal tuberculosis are usually similar to laryngeal carcinoma in most of the cases. ${ }^{4,5}$ We present a case of laryngeal tuberculosis in a 26 year old male who was clinically presented with symptoms of a laryngeal carcinoma without any pulmonary involvement.

\section{Case report:}

A 26 year old male patient presented with hoarseness of voice, dysphagia and odynophagia for five months. There was no history of cough or expectoration. He was giving history of loss of appetite. He was a nonsmoker and there was no history of alcohol or drug abuse. Systemic examination was normal. There was no evidence of cervical lymphadenopathy or discharging sinuses in the neck. Indirect laryngoscopy revealed diffuse soft tissue thickening involving epiglottis, aryepiglottic folds, true and vocal cords with edema along with decreased mobility of the right vocal cord. Chest radiograph and abdominal ultrasonography were normal. Laboratory investigations were within normal limits. He was negative for acid-fast bacilli. Laryngeal carcinoma was suspected for which computed tomography (CT) scan was advised.

Axial CT scan (Figure 1a and 1b) revealed mildly enhancing soft tissue thickening involving epiglottis, aryepiglottic folds, true and vocal cords predominantly on the right side, associated with irregular thickening of the posterior laryngeal wall causing mild narrowing of the laryngeal airway. 

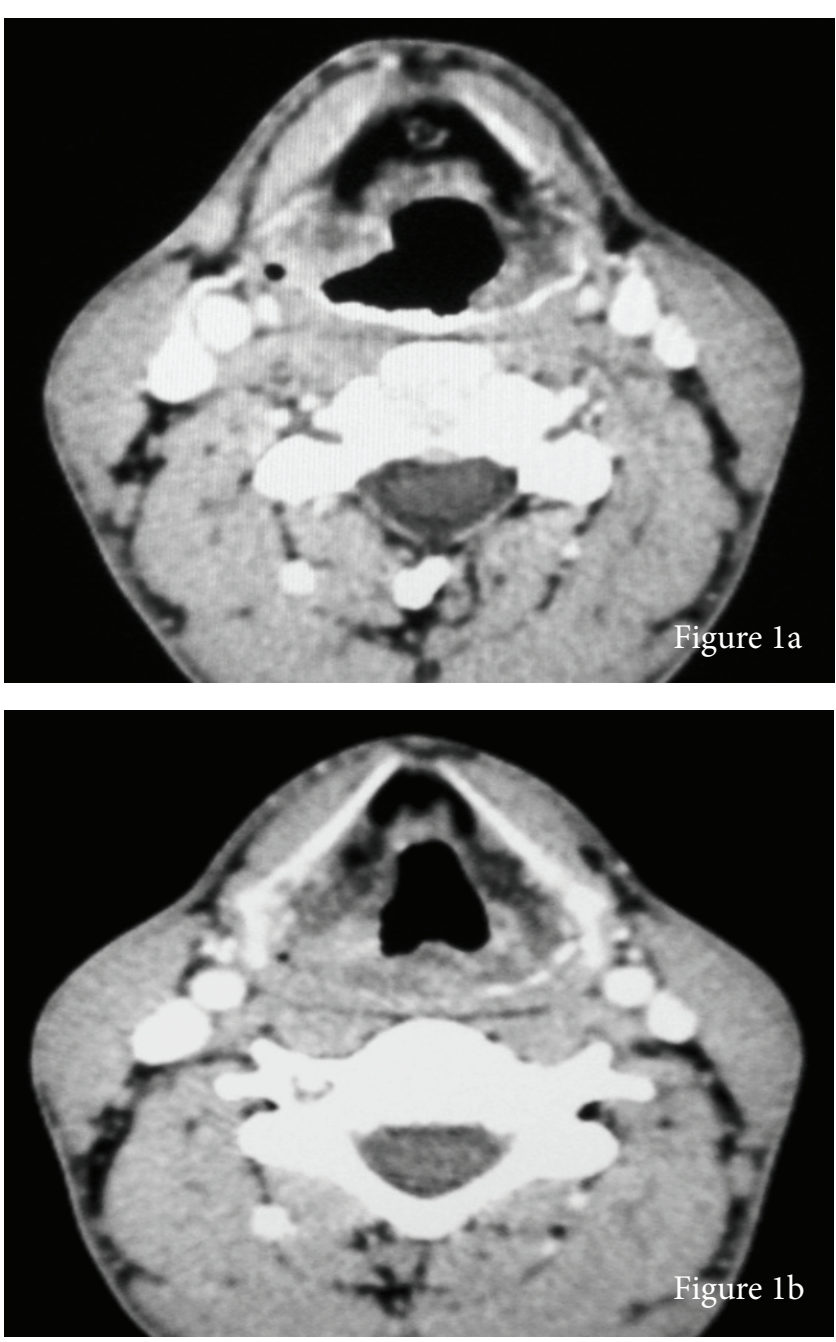

Figure 1a and 1b: Axial section CT image showing soft tissue thickening involving epiglottis, aryepiglottic folds, true and vocal cords with irregular thickening of the posterior laryngeal wall.

The anterior commissure and subglottic region were normal. Laryngeal cartilaginous structures were normal. Biopsy revealed caseating granulomas with lymphocytes, suggestive of laryngeal tuberculosis. Antitubercular therapy was started and patient responded to the treatment.

\section{Discussion:}

Tuberculosis in the head and neck region (extrapulmonary) most frequently occurs in the cervical lymph nodes followed by the larynx..$^{6,7}$ Laryngeal TB is mostly seen in patients of 50-60 years age group, presenting with hoarseness in $80 \%$ to $100 \%$ of cases. Other symptoms are odynophagia, dysphagia, dyspnoea, stridor, cough and haemoptysis. Systemic symptoms are less commonly seen in this disease. ${ }^{4,5}$ Infection to larynx occurs either by a direct spread from the lungs or by haematogenous spread from sites other than the lungs. ${ }^{4,8}$ The distinction between laryngeal TB and chronic laryngitis or laryngeal carcinoma is difficult. Odynophagia is described as an important discriminating symptom, since it is considered rare in laryngeal cancer. True vocal cords are most frequently affected by laryngeal TB, followed by the epiglottis, false vocal cords and ventricles, arytenoids, posterior commissure and the subglottic area. ${ }^{8}$

Radiological findings of laryngeal TB depend on the stage and lesion extension. Infiltrative stage shows focal thickening. Ulcerative stage shows superficial ulcers and rarely reaches the paraglottic spaces and cartilage, perichondritis is seen sometimes, calcification is uncommon and paralaryngeal fat spaces are usually spared. The last stage is characterized by sclerosis.

CT findings in laryngeal TB are edema alone, ulceroinfiltrative mass, infiltrative and pseudo-tumoral appearance; subglottic laryngitis; diffuse form; and tuberculoma. ${ }^{2,9}$ Laryngeal carcinoma is the main differential diagnosis. It is usually not possible to distinguish between these two conditions on imaging. Other differential diagnosis includes laryngeal carcinoma, chronic laryngitis, cat-scratch disease, syphilis, sarcoidosis, Wegener's granulomatosis and fungal infections. ${ }^{6}$

Laboratory investigations include histopathological tissue examinations with Ziehl-Neelsen staining for acid-fast bacilli and identification of mycobacterium tuberculosis by polymerase chain reaction (PCR) or culture. Antitubercular therapy (ATT) is the primary treatment for laryngeal TB. There will be regression of dysphagia within several weeks. ${ }^{6,8}$ If not treated early, laryngeal TB can result in glottis/ subglottic stenosis and vocal cord paralysis if recurrent laryngeal nerve is involved. ${ }^{4,8}$ In laryngeal stenosis, surgery is indicated. ${ }^{9}$

\section{Conclusion:}

This case has been reported to raise awareness of this rare disease. Laryngeal TB should be considered as a differential diagnosis in any laryngeal disease especially laryngeal carcinoma as there are no pathognomonic features indicative of laryngeal TB and it can simulate others lesion. Imaging in correlation to histopathology should be done for diagnosis.

\section{Conflict of interest: None}

\section{References:}

1. Oghan EF, Alper M. Epigllottic tuberculosis in a patient treated with steroids for addissions disease. Tohoku J Exp Med 2003;2:119-25.

2. El Kettani NE, El Hassani MR, Chakir N, et al. Primary 
laryngeal tuberculosis mimicking laryngeal carcinoma: CT scan features. Indian J Radiol Imaging 2010;20:112.

3. Sa LC, Meirelles RC, Atherino CC, et al. Laryngopharyngeal Tuberculosis. Braz J Otorhinolaryngol 2007;73:862-6.

4. Lim JY, Kim KM, Choi EC, et al. Current clinical propensity of laryngeal tuberculosis: review of 60 cases. Eur Arch Otorhinolaryngol 2006;263:838-42.

5. Shin JE, Nam SY, Yoo SJ, et al. Changing trends in clinical manifestations of laryngeal tuberculosis. Laryngoscope 2000;110:1950-3.
6. Moon WK, Han MH, Chang KH, et al. CT and MR imaging of head and neck tuberculosis. Radiographics 1997;17:391-402.

7. Nalini B, Vinayak S. Tuberculosis in ear, nose, and throat practice: its presentation and diagnosis. Am J Otolaryngol 2006;27:39-45.

8. Yencha MW, Linfesty R, Blackmon A. Laryngeal tuberculosis. Am J Otolaryngol 2000;21:122-6.

9. Aspestrand F, Kolbenstvedt A, Boysen M. CT findings in benign expansions of the larynx. J Comput Assist Tomogr 1989;13:222-5. 